\title{
WiP Abstract: Supporting Coordinated Negotiation in CPS Design
}

\author{
X. Sharon Hu, Shengyan Hong, Michael Lemmon \\ University of Notre Dame, Notre Dame, IN 46556, \{shu, shong3, lemmon\}@nd.edu
}

\begin{abstract}
Systematic coordination between the physical world and computation is critical for Quality of Service in the control system. We propose a novel task model to support synergy between the control and real-time sides in the event-trigger control system under environmental perturbations.
\end{abstract}

\section{Motivation}

In cyber-physical system (CPS) design, it is critical to systematically exploit the interplay between the control strategies and the implementation alternatives, such as the intelligent coordination presented in [1], [3], [5]. Negotiation between the two sides could be facilitated through appropriately choosing task models and dynamically managing task execution. Specifically, desired performance of the control plants shape the choices of the parameters for a specific real-time task model, while the tasks based on the task model need be scheduled in the cyber side.

In real-time systems, there are three well known task models, i.e., the periodic task model, the sporadic task model, and the aperiodic task model. The models are either too constraining or too general for many cyber-physical systems. For example, consider the control task for a vehicle's anti-lock brake system. Under normal situations, the task is executed periodically with a relatively large period. As soon as the wheels are detected to be locked, the control task needs to be executed much more frequently. However, this frequency can be gradually reduced when the situation improves. It is obvious that the aforementioned task models would either require much higher resource than necessary or cannot offer the desired Quality of Service.

The elastic task model [2], [3] may be able to model the above system, because task periods can be any value within a given range. However, the choices of the actual period values are made based on satisfying the timing requirement instead of in response to the physical system's requirement. Furthermore, the elastic task model is fundamentally still a periodic task model except that task periods can be adjusted once in a while.

\section{Proposed Solution}

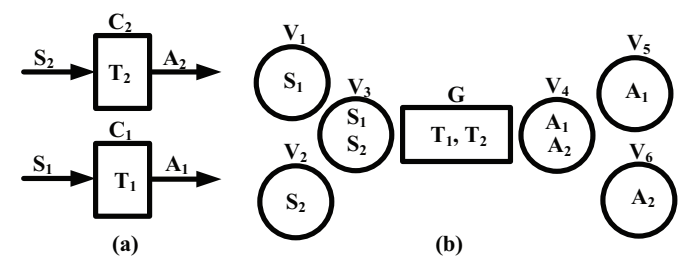

Fig. 1. An example control loop set and its routing topology

We propose a new task model that aims to connect directly the control system behavior with real-time task modeling. This model assumes that the separation interval (i.e., task period) and the maximum allowed delay (i.e., task deadline) are defined as functions of time and external disturbances to the physical system. In addition, the model may allow the system to trade off task execution times with task periods and deadlines. In this way, the dynamics of the control system can be reflected in the task model and be exploited to improve resource utilization.

In our preliminary work, we have adopted this task model in a networked control system that is similar to WirelessHART [4]. The system contains a gateway (where the control algorithms are evaluated), a set of sensor nodes, a set of actuator nodes, and a set of relay nodes. The gateway and all the nodes maintain an existing schedule for nominal situations. When disturbances demand changes to the timing parameters in the task model for some control tasks, the existing schedule needs to be adjusted timely by the gateway to avoid packet drops. Since totally redesigning the schedule is too expensive, we implement an online re-negotiation strategy for the network in response to the perturbation in a distributed and low-cost manner. This re-negotiation strategy is built on the aforementioned new task model.

As a simple example shown in Figure 1, we consider two control loops $C_{1}$ and $C_{2}$. Each control loop $C_{i}$ is composed of a sampling stream $S_{i}$, a control task $T_{i}$, and an actuator stream $A_{i}$, as shown in Figure 1(a), while the routing topology of the loops are shown in Figure 1(b). The new task model not only reflects how the task parameters will change in the near future, but also help packet schedules be adjusted properly. We have used realistic control parameters in the new task model and analyzed the system timing behavior for different packet schedules. Our results reveal that re-negotiation based on the new task model is very effective in handling dynamic changes to the timing parameters of the control tasks.

\section{FUTURE WORK}

We are developing new resource management scheme based on the changes to the parameters of control tasks, which are provided by the new task model, in order to achieve required performance of control applications.

\section{REFERENCES}

[1] S. Bak, K. Manamcheri, S. Mitra, and M. Caccamo, "Sandboxing controllers for cyber-physical systems," in ICCPS, 2011.

[2] G. C. Buttazzo, G. Lipari, M. Caccamo, and L. Abeni, "Elastic scheduling for flexible workload management," IEEE Trans. Computers, vol. 51, no. 3, pp. 289-302, 2002.

[3] T. T. Chantem, X. S. Hu, and M. D. Lemmon, "Generalized elastic scheduling for real-time tasks," IEEE Trans. Comput., vol. 58, no. 4, 2009.

[4] D. Chen, M. Nixon, and A. Mok, "Wirelesshart: real-time mesh network for industrial automation." Springer, 2010.

[5] T. Yoshimoto and T. Ushio, "Optimal arbitration of control tasks by job skipping in cyber-physical systems," in ICCPS, 2011. 